\title{
Nucleotide-binding oligomerization domain containing-like receptor family, caspase recruitment domain (CARD) containing 4 (NLRC4) regulates intrapulmonary replication of aerosolized Legionella pneumophila
}

\author{
William R Berrington*, Kelly D Smith, Shawn J Skerrett and Thomas R Hawn
}

\begin{abstract}
Background: Legionella pneumophila (Lp) flagellin activates signaling pathways in murine macrophages that control Lp replication. Nucleotide-binding oligomerization domain (NOD) containing-like receptor (NLR) family, caspase recruitment domain (CARD) containing 4 (NLRC4) and Toll-like Receptor (TLR5) both recognize Lp flagellin in vitro, but whether these two receptors play redundant or separate functional roles in vivo is unknown.

Methods: The immune response of N/rc4-/-, N/rc4-/-/T/r5-/-, and wild type C57BI/6 mice was analyzed after in vivo infection with aerosolized Lp.

Results: Lp clearance from the lungs was delayed in N/rc4-/- mice over seven days in comparison to wild type controls. NIrc4-/-/TIr5-/- mice had no additional defect. In contrast to TLR5, NLRC4 did not regulate recruitment of neutrophils to the lung. Although there were no differences among the mouse strains in the lung transcriptome at 4 hours, N/rc4-/- and N/rc4-/-T/r5-/- mice had increased lung inflammation at 72 hours in comparison to WT. Nirc4-/-/T/r5-/- mice also had altered cytokine production at both 4 and 24 hours post infection when compared to wild-type (WT) and N/rc4-/- mice. Lp replication in murine alveolar macrophages was NLRC4-dependent and TLR5-independent.
\end{abstract}

Conclusion: These studies reveal that NLRC4 and TLR5 mediate different roles in the inflammatory response to Lp flagellin in an aerosolized infection model and NLRC4 regulates replication in both lungs and alveolar macrophages.

Keywords: Legionella pneumophila, Pneumonia, Alveolar macrophage, NLRC4, TLR5

\section{Background}

Legionella pneumophila (Lp), an intracellular gramnegative rod, is a frequent cause of severe pneumonia [1]. Lp replicates and survives in the environment by infecting and persisting in waterborne amoeba and human infection occurs following exposure to aerosols of contaminated water [2]. Despite the discovery of Lp over three decades ago, we are just beginning to understand the role that innate recognition of molecules such as flagellin plays in host defense to the pathogen.

\footnotetext{
* Correspondence: berring@uw.edu

Department of Medicine, University of Washington School of Medicine, 1959 NE Pacific Street, Box 35642398195-6523, Seattle, Washington, USA
}

Lp is recognized by several classes of innate immune receptors that regulate different steps in the immune response. Extracellular and endosomal receptors (Toll-like receptor (TLR)-4, TLR2, TLR9 and TLR5) and cytoplasmic receptors, NLRC4, Neuronal apoptosis inhibitory protein-5 (NAIP5), Retinoic acid inducible gene - I, Melanoma differentiation associated protein-5, NLR family, pyrin domain containing-7 (NLRP7), NOD1, and NOD2) detect Lp in the mouse and regulate the immune response to the pathogen [3-13]. Two receptor complexes, TLR5 and NLRC4/NAIP, detect Lp flagellin (FlaA) [4,14-16]. Through a series of in vitro studies of Lp replication in murine bone marrow derived 
macrophages (BMDM), NAIP5 was identified as a key regulator of bacterial growth $[17,18]$. Later studies showed that NAIP5, NLRC4, and apoptosis-associated speck protein containing CARD (ASC) oligomerize into an inflammasome complex after recognition of cytoplasmic flagellin leading to enhanced caspase- 1 dependent IL-1 $\beta$ secretion and pyroptotic cell death $[10,11,14,16]$. In addition in the mouse Lp flagellin is detected by multiple NAIP orthologs (NAIP5 and NAIP6), causing oligomerization of the NLRC4 inflammasome and proteolytic activation of caspase-1 $[19,20]$. While replication of $\mathrm{LP}$ in cultured murine BMDM is controlled through activation of the NLRC4/NAIP inflammasome, the role of the inflammasome in mediating resistance to Lp in alveolar macrophages and in the lungs of whole animals is only partially understood. Respiratory challenge studies with wild type and flagellin-deficient (FlaA-) Lp indicated that flagellin recognition is important for control of Lp replication in the lungs $[4,15,21,22]$. The A/J mouse, which has impaired signaling through the NLRC4/NAIP5 inflammasome, permits intrapulmonary replication of Lp, in contrast to mice with intact NLRC4 inflammasome activation [23]. In addition, pulmonary clearance of Lp is impaired in Nlrc4-/- mice, a finding that is reproduced by flagellin deficient organisms $[21,22]$. Therefore we know that the NLRC4/NAIP5 inflammasome helps to clear Lp from the lung, but the mechanism of clearance is largely unknown, and whether other flagellin detectors such as TLR5 play a redundant role also is unknown.

FlaA expression in $\mathrm{Lp}$ is a major virulence factor and important in the early host response to the organism $[15,24]$. Lp tightly controls the expression of flagellin to enhance its infectivity and suppression of flagellin expression is important in intracellular replication in the macrophage $[25,26]$. Most experiments identifying flagellin as an important determinant in macrophages, however, have been performed in bone marrow derived macrophages differentiated ex vivo, and the role that tissue macrophages such as the alveolar macrophage play in control of Lp replication in vivo remains largely unknown.

In this study we examine the in vivo immune response to aerosolized Lp infection in Nlrc4-/- and Nlrc4 $-/-/$ Tlr5-/- mice to determine whether impaired signaling through two separate flagellin detection pathways influences host immune responses and Lp survival in vivo. In addition we identify that alveolar macrophages restrict Lp growth in an NLRC4-dependent, but not TLR5-dependent manner.

\section{Methods \\ Bacteria}

L. pneumophila Corby (serogroup 1) and L. pneumophila Corby flagellin deficient $(\Delta$ flaA) were gifts from
K Heuner [26]. L. pneumophila Philadelphia-1 was obtained from the American Type Culture Collection (ATCC 33152).

\section{Animal model}

Tlr5-/- and Nlrc4-/- C57Bl/6 mice strains were obtained from Drs. Shizuo Akira and Vishva Dixit, respectively $[27,28]$, and were backcrossed at least six generations onto C57BL/6. Wild type C57BL/6 mice were purchased from the Jackson Laboratory (Bar Harbor, ME). Mice were housed in laminar flow cages with ad lib access to sterile food and water. Male and female mice were used in approximately equal numbers, and were 8-10 weeks of age at the time of experimentation. The University of Washington Institutional Animal Care and Use Committee approved all experiments.

\section{Airborne infection model}

Mice were exposed to aerosolized bacteria in a whole animal exposure chamber as described previously [4,29]. Lp was cultured on buffered yeast charcoal extract (BYCE) agar plates for 4 days at $35^{\circ} \mathrm{C}$. Colonies were harvested by rinsing plates with PBS. The pooled suspension was pelleted by centrifugation and resuspended in PBS to approximately $3 \times 10^{10}$ cells $/ \mathrm{ml}$, as estimated by optical density. The slurry was transferred to twin jet nebulizers from Salter Laboratories (Arvin, CA USA) and mice were exposed to aerosolized bacteria for $30 \mathrm{mi}-$ nutes. To determine actual bacterial deposition in the lungs in each experiment, four sentinel animals were euthanized with intraperitonal pentobarbital and exsanguinated immediately after aerosol exposure. Left lungs were homogenized in PBS and serial dilutions in Mueller Hinton broth were plated onto BYCE agar. At subsequent time points, left lungs were similarly homogenized and cultured. Unused lung homogenates were mixed in lysis buffer containing protease inhibitors (Roche, NJ) on ice for 30 minutes, centrifuged at 2,500 rpm, and stored at $-80^{\circ} \mathrm{C}$ for further use. Right lungs were used for histology and alveolar cell recruitment, as described [4,30]. Following cannulation of the trachea, right lungs were lavaged four times with $0.5 \mathrm{ml}$ volumes of $0.9 \%$ sodium chloride containing $0.6 \mathrm{mM}$ EDTA. Cell counts in bronchoalveolar lavage samples were determined with a hemocytometer, and differentials were determined by examining cytocentrifuge specimens stained with a modified Wright-Giemsa technique (Diff Quick, Dade Behring, Dudingen, Switzerland). After lavage, right lungs were fixed by instillation of $4 \%$ paraformaldehyde at $20 \mathrm{~cm}$ of pressure. Sections of paraffin-embedded tissue were stained with hematoxylin and eosin. 20 separate high power fields of lung sections were examined by a pathologist and scored to determine the percentage of airspaces with inflammation that involved the alveolar airspaces. 


\section{Protein detection}

Lung homogenate cytokine levels were determined using a multiplex fluorescent bead array system (Luminex 100). Briefly dilutions of homogenates were captured onto antibody-coated fluorochrome embedded microspheres and read using a flow based sorting and detection platform. Cytokine levels were measured using a sandwich ELISA technique (R\&D systems) as previously described $[4,30]$.

\section{Alveolar macrophage isolation and infection}

Resident alveolar macrophages (AMs), were harvested from uninfected mice as previously described [4]. Cells pooled from 8-10 mice were suspended in RPMI 1640 supplemented with $10 \%$ heat-inactivated FCS, $100 \mathrm{U} / \mathrm{ml}$ penicillin, $100 \mathrm{ug} / \mathrm{ml}$ streptomycin, and $2 \mathrm{mM}$ L-glutamine, counted in a hemocytometer, and the viability was determined by the exclusion of trypan blue. The cells were cultured in poly-L-lysine-treated 96 well plates at a density of $1 \times 10^{6}$ viable $\mathrm{AM} / \mathrm{ml}(0.85 \mathrm{ml} /$ well $)$. After a $2 \mathrm{~h}$ incubation at $37^{\circ} \mathrm{C}$ in humid air with $5 \% \mathrm{CO}_{2}$, the adherent monolayers were washed five times with prewarmed Hank's balanced salt solution (HBSS) and re-fed with antibiotic-free medium. Lp was added at an MOI of 1 and the plates were centrifuged at $400 \times$ g for 7 minutes, then incubated for 1 hour. The monolayers were washed 4 times with warm HBSS and re-fed with antibiotic-free medium. Immediately and after 24,48 , and $72 \mathrm{~h}$ incubation at $37^{\circ} \mathrm{C}$ in humid air with $5 \% \mathrm{CO}_{2}$ triplicate monolayers were lysed by the addition of saponin to a concentration of $0.1 \%$. The lysate was serially diluted in Mueller Hinton broth and quantitatively cultured on buffered charcoal yeast extract (BCYE) agar. Colonies were counted after 3 days of incubation at $37^{\circ} \mathrm{C}$ in $5 \% \mathrm{CO}_{2}$.

\section{Lung RNA isolation}

Mice exposed to aerosolized Lp $4 \mathrm{~h}$ previously and uninfected controls were euthanized with pentobarbital and exsanguinated by cardiac puncture. The pulmonary vascular bed was perfused with $10 \mathrm{ml}$ cold PBS and both lungs were harvested into RNALater (Qiagen, Hamburg, Germany). Total lung RNA was isolated with an RNAeasy column (Qiagen, Hamburg, Germany). Reverse transcriptase was performed with Superscript III reverse transcriptase (Invitrogen, Carlsbad, CA) according to the manufacturer's protocol. Total lung cDNA was applied to murine exon arrays (Affymetrix 430 2.0) (Affymetrix, Santa Clara, CA) containing 45,100 probes for 22,006 genes. Each probe was analyzed separately. Induced genes in lung mRNA were determined by comparing the expression values of the probes (which were analyzed separately) in infected and uninfected mice. Genes induced in infected wild type $\mathrm{C} 57 \mathrm{Bl} / 6$ mice $(\mathrm{n}=2)$ were compared to genes from infected in Nlrc4-/mice $(\mathrm{N}=2$ each) as well as uninfected C57Bl/6 $(\mathrm{n}=2)$ and Nlrc4-/- $(\mathrm{n}=2)$ mice. Raw intensity values were background corrected, $\log 2$ transformed and quantile normalized and adjusted by robust multichip average (RMA) prior to analysis [31].

\section{Statistics}

Data are expressed as mean \pm standard error of mean (SEM). For comparisons of continuous variables among multiple groups, significance was determined using oneway ANOVA with bonferroni post-hoc test analysis. Comparisons between two groups was analyzed with two-tailed $\mathrm{T}$ tests. For mRNA expression arrays, a generalized linear model for microarray by limma (Bioconductor [32]) was used. Unadjusted significance was determined for expression arrays by an empirical Bayes moderated t-statistic as described [33] using the equations: (1) (WTstimulatedWTunstimulated)-(Nlrc4-/-stimulated - Nlrc4-/-unstimulated), (2) (WTstimulated-WTunstimulated), and (3) (Nlrc4-/-stimulated - Nlrc4-/-unstimulated). For adjusted $\mathrm{p}$ values, a false discovery rate of $5 \%$ was used for the analysis in the manner described by Benjamini and Hochberg [34]. The cutoff of significance for genes included in Additional file 1: Table S1 and Additional file 2: Table S2 was an adjusted P value of 0.01 , no cutoff for $\log _{2}$ fold change was used. Gene set enrichment was done using publicly available software (Broad institute $[35,36]$ ), using a false discovery rate of $25 \%$.

\section{Results}

\section{NLRC4 regulates pulmonary clearance of Lp, but not} cellular recruitment to the lungs

We infected WT, Nlrc4-/-, and Tlr5-/-Nlrc4-/- mice with aerosolized Lp to determine which immune responses are regulated by NLRC4 and TLR5 during pneumonic infection. Nlrc4-/- mice exhibited markedly delayed clearance of Lp from the lungs compared to WT mice; a 25-fold difference in lung CFUs was evident at the 72 hour time point and a 10-fold difference persisted to 10 days after infection (Figure $1 \mathrm{~A}$ and data not shown (for day 10)). Nlrc4-/-/Tlr5-/- mice had no additional impairment in Lp clearance compared to the Nlrc4-/animals (Figure 1A). Interestingly, Lp-infected Nlrc4-/animals showed no difference in lung recruitment of neutrophils at 4, 24, or 72 hours in comparison to WT mice. In contrast, TLR5 and NLRC4 double knockout showed impaired neutrophil recruitment at 4 hours, similar to previously reported findings in Tlr5-/- animals (Figure 1B) [4]. These data demonstrate that NLRC4 is required for optimal clearance of Lp after airborne infection, but does not influence inflammatory cell recruitment to the lungs.

\section{NLRC4 and TLR5 regulate late cytokine production in Lp infected mice}

Next we examined whether TLR5 and NLRC4 regulate cytokine production in the lungs of mice infected with 



Figure 1 NLRC4 regulates Lp replication, but not inflammatory cell recruitment to the lung after aerosolized infection. WT (circles), N/rc4-/- (squares), and N/rc4-/-/T/r5-/- mice (triangles) were exposed to aerosolized Lp Philadelphia-1 by aerosol infection ( $\mathrm{n}=8-12$ for each time point). (A) Lp CFU measured at 4, 24 (1 day), 72 (3 days), and 168 (7 days) hours following infection (B) Lung neutrophil and (C) monocyte counts obtained by bronchoalveolar lavage of the right lung. Combined results from three separate experiments are depicted. Data are mean \pm SEM $+=p<0.01$ and ${ }^{*}=p<0.05$ as determined by one-way ANOVA.

Lp. At $4 \mathrm{~h}$ post-infection, the lungs of Nlrc4-/-/Tlr5-/had decreased levels of tumor necrosis factor (TNF) compared to WT control lungs. These findings, however, were not consistent between two independent experiments (Figure 2E). Conversely at 24 hours post infection, TNF levels were consistently enhanced in Nlrc4-/-/Tlr5-/mice when compared to Nlrc4-/- or WT mice (Figure 2E). In addition Nlrc4-/-/Tlr5-/- double knockout mice had significantly more proinflammatory chemokine (C-X-C motif) ligand 2 (CXCL2 or MIP-2) (Figure 2C) and interleukin 6 (IL-6) (Figure 2F) when compared to Nlrc4-/(for CXCL2) or WT (for CXCL2 and IL-6) mice. No changes in lung levels of Interleukin 1-beta (IL-1 $\beta$ ) (Figure 2A), chemokine (C-X-C motif) ligand 1 (CXCL-1 or $\mathrm{mKC}$ ) (Figure $2 \mathrm{~B}$ ), chemokine (C-C motif) ligand 2 (CCL2 or MCP-1) (Figure 2D), GM-CSF (Figure 2G), and IFN- (Figure $2 \mathrm{H}$ ) were detected at $4 \mathrm{~h}$ and $24 \mathrm{~h}$ when comparing WT, Nlrc4-/-, and Nlrc4-/-/Tlr5-/- mice. We also saw that during the 72 hour timepoint, there were increased cytokines (IL-1 $\beta$, MIP2, CXCL-1, MCP-1, and IL-6) observed in Nlrc4-/-/Tlr5-/- mice (data not shown). Together, these data suggest that animals deficient in both NLRC4 and TLR5 pathways produce higher levels of lung pro-inflammatory cytokines than WT or NLRC4 single knockout animals.

\section{NLRC4 does not regulate early lung transcriptional responses}

The NLRC4 inflammasome activates caspase- 1 and also influences macrophage survival. However, little is known if genes downstream of NLRC4 control replication in the lungs during in vivo infection. To explore this, we isolated mRNA from the lungs of Lp-infected WT and Nlrc4-/- mice 4 hours after infection $(\mathrm{n}=2)$ and measured expression levels with an Affymetrix 4302.0 exon array. 1701 probes from 1275 genes were differentially regulated (972 probes were induced and 729 repressed) in the WT mice and 1315 probes from 978 genes (980 probes were induced and 335 repressed) in Nlrc4-/animals compared to the uninfected controls (using a linear model fit from Bioconductor, $\mathrm{p}<0.01$ [33,37]) (Figure 3, Table 1, Additional file 1: Table S1, Additional file 2: Table S2, Additional file 3: Table S3, and Additional file 4: Table S4). When comparing infected animals, only 4 probes from 3 genes (D site albumin promoter binding protein, RIKEN cDNA G530011006 gene, testis expressed gene 11) were different between WT and Nlrc4-/- mice (Table 1 , using the formula $\left[\left(\mathrm{WT}_{\text {stim }}-\mathrm{WT}_{\text {unstim }}\right)\right.$ (Nlrc4-/- stim $-N l r c 4-/$ unstim $_{\text {) }}$ ] with a generalized linear model with a false discovery rate of $<5 \%$ ) (Table 1 ). In addition gene set enrichment analysis (GSEA) was performed using a $25 \%$ false discovery rate cutoff and no gene sets were noted to be significantly different in the lungs of Nlrc4-/- mice compared to WT after infection with LP. Together, these data suggest that the whole lung transcriptional response to Lp is very similar between WT and Nlrc4-/- mice.

\section{NLRC4 regulates lung inflammation}

We next examined lung histology in WT, Nlrc4-/-, and Nlrc4-/-/Tlr5-/- mice at 1 and 3 days after infection and determined the percentage of inflamed airspace (Figure 4A). No difference was seen between WT and Nlrc4-/-, and Nlrc4-/-/Tlr5-/- mice at 24 hours following aerosol inoculation (Figure 4A and B). At 3 days following inoculation, WT lungs showed significantly less inflammation then the Nlrc4-/- and Nlrc4-/-/Tlr5 -/- animals (Figure 4B). Compared to WT mice, the Nlrc4-/- and Nlrc4-/-/Tlr5-/- mice had increased patchy infiltration of the alveolar space by neutrophils with some macrophages, as is characteristic for Lp pneumonia in humans. No significant difference was seen between Nlrc4-/- and Nlrc4-/-/Tlr5-/- mice. These data suggest that increased replication in Nlrc4-/- mice is associated with an increased inflammatory response in the lungs 3 days following Lp exposure. 

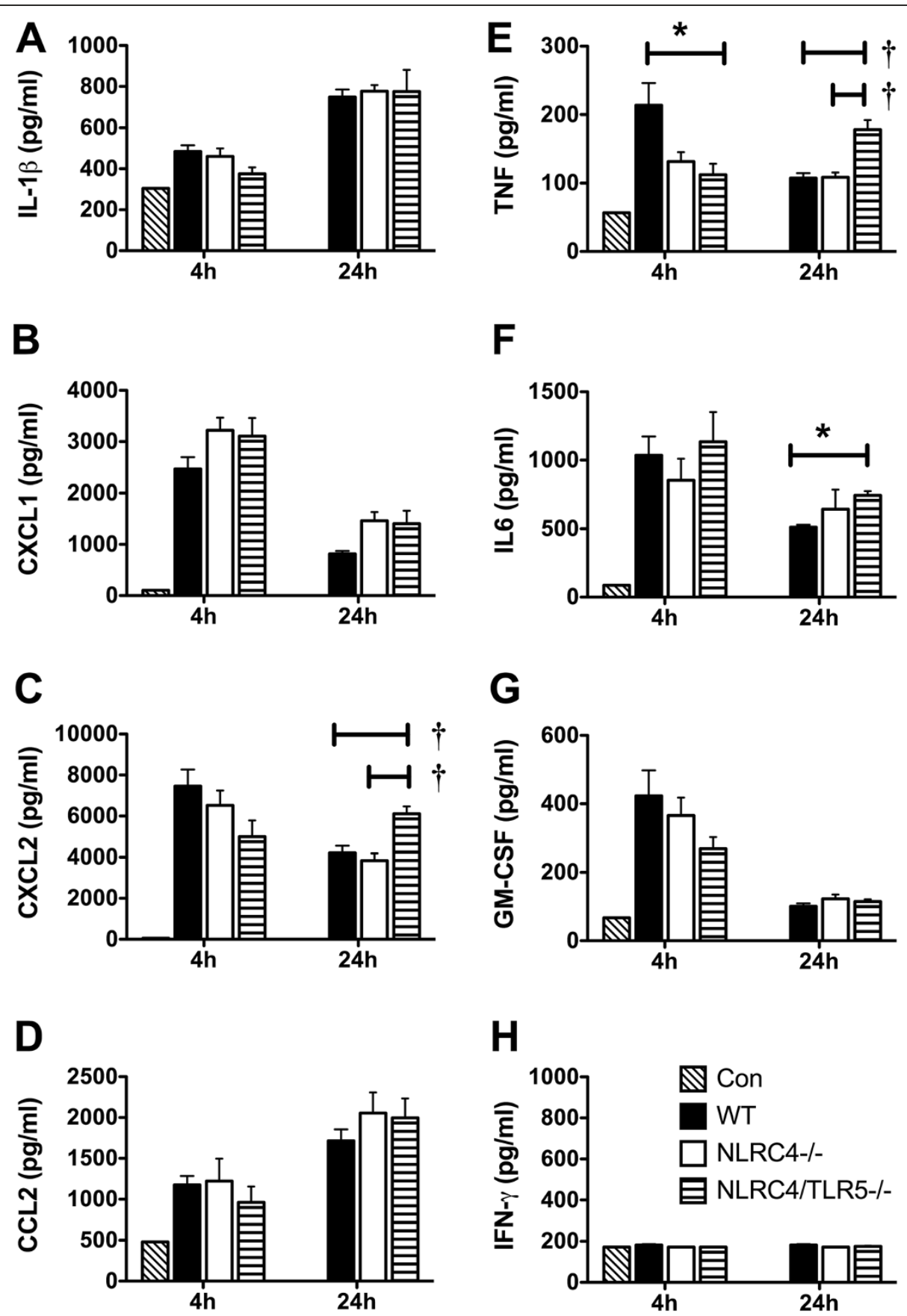

Figure $\mathbf{2}$ Lung cytokine levels in mice infected with aerosolized $L$. pneumophila. Cytokine levels in lung homogenates of mice infected with aerosol infection by Lp Phil-1. IL-1ß (A), CXCL1 (B), CXCL2 (C), CCL2 (D), TNF (E), IL-6 (F), GM-CSF (G), and IFN- $\mathbf{( H )}$ were measured by fluorescent bead immunoassay at 4 hours and 24 hours in untreated controls (diagonal hatched bars), WT (C57/Bl6) (black bars), N/rc4-/- (white bars), and N/rc4-/-/T/r5-/- (horizontal hatched bars) mice. Cytokine levels from uninfected controls are shown in (Con) lane at 4 h. Data is from $\mathrm{n}=4-8$ mice except uninfected control $(\mathrm{n}=1) .{ }^{*}=\mathrm{p}<0.05, \dagger=\mathrm{p}<0.01$ as determined by one-way ANOVA test.

NLRC4, but not TLR5, controls intracellular replication in alveolar macrophages

To examine possible mechanisms of NLRC4-dependent Lp replication in lungs, we next measured Lp replication in alveolar macrophages. We infected WT, Nlrc4-/- and Tlr5-/- alveolar macrophages with LpWT or LpFlaA- and examined replication over 72 hours. Murine alveolar macrophages restricted LpWT growth (Figure 5) in a NLRC4, but not TLR5, dependent manner (Figure 5 A-C). Alveolar macrophages harvested from WT and TLR5-deficient mice supported replication of LpFlaAbut not LpWT, whereas both Lp strains replica- 


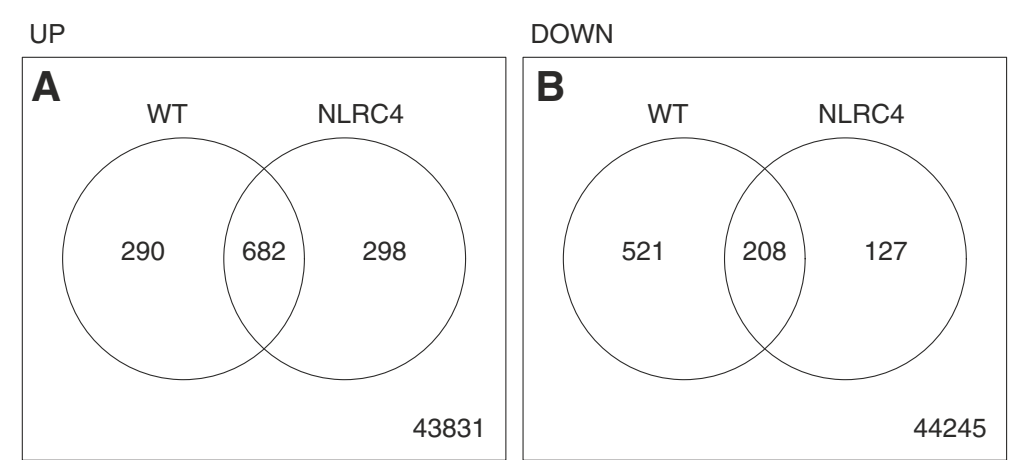

Figure 3 Lung mRNA transcriptome after aerosolized Lp infection. Whole lung RNA was isolated 4 hours following Lp infection of WT and N/rc4-/- mice and analyzed for differential expression compared to uninfected controls by exon array analysis. 2 mice were analyzed per condition. Venn diagrams of genes increased (A) and decreased (B) compared to uninfected controls are depicted. Significantly different genes induced or suppressed by Lp infection in WT and N/rc4-/- lungs are listed in Additional file 1: Table S1 and Additional file 2: Table S2 respectively. 3 genes were significantly increased or decreased in WT versus N/rc4-/- lungs (Table 1). Significant difference in genes was determined by general linear model using a false discovery rate of 0.05 (see methods).

Table 1 Genes significantly different between WT and NIrc4-/- Lp-infected mice

\begin{tabular}{|c|c|}
\hline Genes different between WT and NIrc4-/- mice & Name \\
\hline Dbp & D site albumin promoter binding $\mathrm{p}$ \\
\hline Tex11 & Testis expressed gene 11 \\
\hline Dbp & D site albumin promoter binding $p$ \\
\hline G530011006Rik & RIKEN cDNA G530011006 gene \\
\hline \multicolumn{2}{|c|}{ Genes different between WT infected and uninfected mice } \\
\hline Slc26a4 & Solute carrier family 26 , member 4 \\
\hline Cd14 & CD14 antigen \\
\hline Cxcl1 & Chemokine (C-X-C motif) ligand 1 \\
\hline Ereg & Epiregulin \\
\hline Gm1960 & Gene model 1960, (NCBI) \\
\hline Cxcl1 & Chemokine (C-X-C motif) ligand 1 \\
\hline Steap4 & STEAP family member 4 \\
\hline G530011006Rik & RIKEN cDNA G530011006 gene \\
\hline$\| 4 i_{1}$ & Interleukin 4 induced 1 \\
\hline $\mathrm{CxCl} 2$ & Chemokine (C-X-C motif) ligand 2 \\
\hline
\end{tabular}

$$
\begin{aligned}
& \text { Fold change (95\% Cl), } p \\
& 0.05(0.04-0.05), p=6.62 E-03 \\
& 0.19(0.16-0.21), p=2.65 E-03 \\
& 0.04(0.03-0.04), p=5.62 E-04 \\
& 46.41(39.30-54.80), p=2.51 E-03 \\
& 52.83(46.51-60.02), p=1.37 E-05 \\
& 15.25 \text { (13.64-17.06), } p=1.58 E-05 \\
& 166.48(147.58-187.81), p=1.73 E-05 \\
& 11.31(10.11-12.64), p=2.07 E-05 \\
& 383.16(331.67-442.63), p=2.07 E-05 \\
& 56.19(49.78-63.43), p=2.07 E-05 \\
& 14.71(13.02-16.63), p=2.07 E-05 \\
& 72.30(61.82-84.56), p=2.48 E-05 \\
& 41.87(33.79-51.89), p=3.09 E-05 \\
& 399.39(355.05-449.26), p=3.32 E-05
\end{aligned}
$$

Genes different between NIrc4-/- infected and uninfected mice

$\begin{array}{lll}\text { Tbl3 } & \text { Transducin (beta)-like } 3 & 2.09(1.85-2.37), p=2.93 \mathrm{E}-03 \\ \text { Gja1 } & \text { Gap junction membrane channel protein alpha 1 } & 2.50(2.22-2.81), p=6.04 \mathrm{E}-04 \\ \text { Gja1 } & \text { Gap junction membrane channel protein alpha 1 } & 3.39(3.04-3.78), p=3.75 \mathrm{E}-04 \\ \text { Slc16a1 } & \text { Solute carrier family 16 (monocarboxylic acid } & 1.53(1.29-1.80), p=8.34 \mathrm{E}-03 \\ & \text { transporters), member 1 } & \\ \text { Sfrs2 } & \text { Splicing factor, arginine/serine-rich 2 (SC-35) } & 2.06(1.79-2.37), p=9.27 \mathrm{E}-04 \\ \text { Scd2 } & \text { Stearoyl-Coenzyme A desaturase 2 } & 0.55(0.49-0.63), p=3.51 \mathrm{E}-03 \\ \text { Scd2 } & \text { Stearoyl-Coenzyme A desaturase 2 } & 0.48(0.43-0.54), p=2.79 \mathrm{E}-03 \\ \text { Aldh18a1 } & \text { Aldehyde dehydrogenase 18 family, member A1 } & 1.86(1.66-2.08), p=6.23 \mathrm{E}-03 \\ \text { Junb } & \text { Jun-B oncogene } & 5.19(4.66-5.79), p=2.32 \mathrm{E}-04 \\ \text { Marcks1 } & \text { MARCKS-like 1 } & 4.94(4.44-5.50), p=2.31 \mathrm{E}-04\end{array}$

Depicted above are the top ten most significant genes differentially expressed in 1) N/rc4-/- vs T/r5-/-, 2) WT infected and uninfected and 3) N/rc4-/- infected and uninfected as determined by a general linear model using a false discovery rate of 0.05 .

Complete gene lists are included in online supplemental material to highlight similarities between probes differentially regulated between WT and NLRC4-/- infected lungs. 


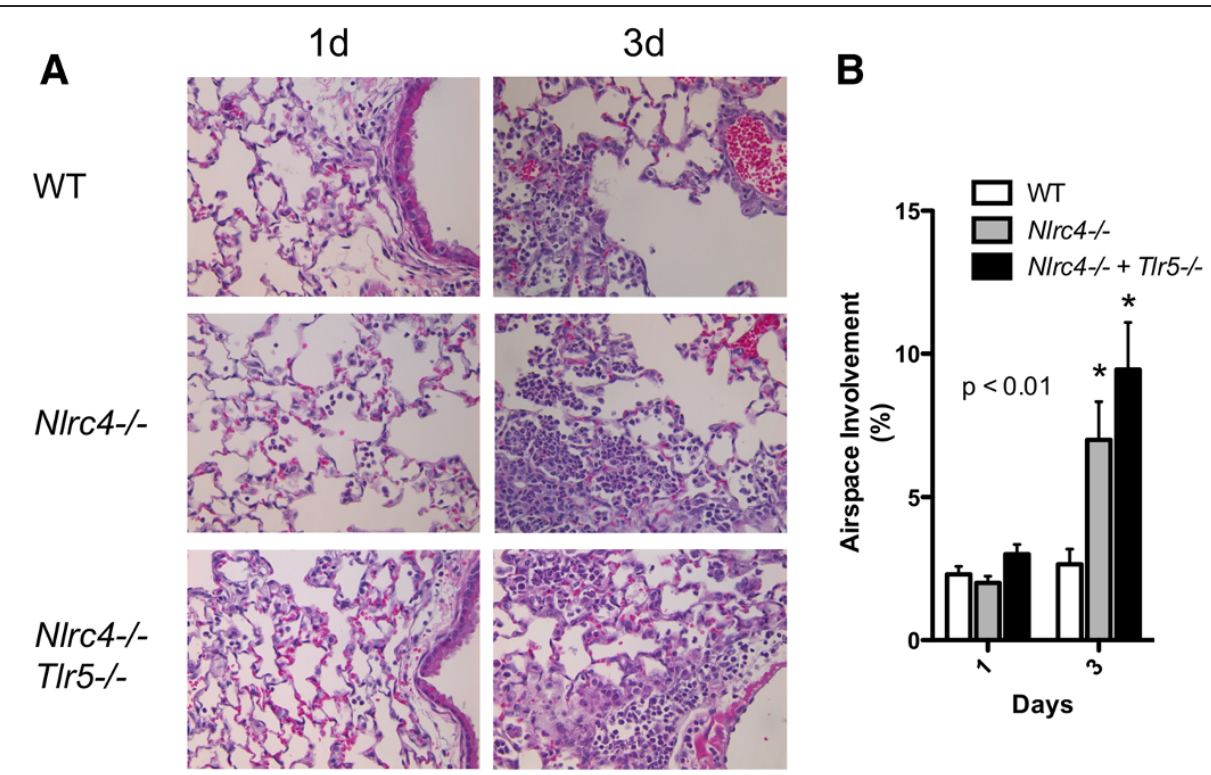

Figure 4 Lung histology in NIrc4-/- and NIrc4-/-/TIr5-/- mice after aerosolized Lp infection. (A) Lungs were isolated from mice infected with aerosolized Lp and stained with Hematoxylin and Eosin. Graphic depiction of inflammation scored by pathologist is shown in (B) $p<0.01$ by 2 way ANOVA for data analysis, scoring 10 separate fields from 2 different lungs from WT (open columns), NIrc4-/- (grey), and N/rc4-/-/T/r5-/- mice.

ted alveolar macrophages from NLRC4-null mice (Figure 5A-C). These data indicate that NLRC4, but not TLR5, restricts the growth of Lp in alveolar macrophages, and suggest that flagellin induces NLRC4mediated resistance to $\mathrm{Lp}$ in murine alveolar macrophages.

\section{Discussion}

Our primary finding is that TLR5 and NLRC4 mediate different roles in the inflammatory response to Lp flagellin in an aerosolized infection model. In earlier studies, TLR5 detection of Lp flagellin was shown to regulate early recruitment of PMNs to the alveolar space that results in organizing pneumonia at later stages without a difference in bacterial replication [4]. In this study, we again observed that TLR 5 regulates early recruitment of PMNs to the alveolar space but not bacterial replication in Nlrc4-/-/Tlr5-/- mice compared to Nlrc4-/- and WT mice. In contrast to TLR5, we show here that NLRC4 regulates bacterial replication in vitro in alveolar macrophages and in vivo by accelerating Lp clearance from the lungs.

NLRC4 control of in vivo Lp growth could be mediated via several mechanisms including pyroptosis or via

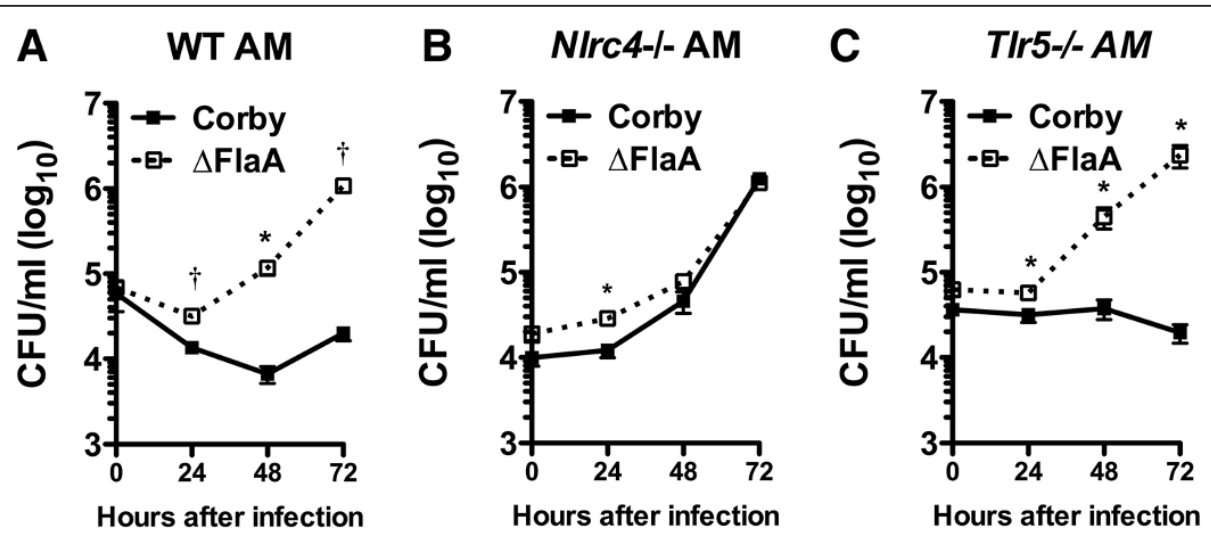

Figure 5 NLRC4 deficiency impairs replication in murine alveolar macrophages. Alveolar macrophages (AM) were isolated from lungs of WT (A), N/rc4-/- (B), and T/r5-/- (C) mice by bronchoalveolar lavage of uninfected mice. Cells were infected ex vivo with WT (Corby) Lp or isogenic FlaA- (Corby) Lp and CFUs were measured in lysed monolayers at 24, 48, and 72 hours. Data are mean \pm SEM of triplicate or quadruplicate monolayers at each time point (except for duplicate measures at time 0 from TLR5-/- mice.). ${ }^{*} p<0.05,+p<0.005$ as determined by two-tailed Students' t-test. 
IL-1 $\beta$ or IL-18-dependent mechanisms. Previous in vitro studies in murine BMDM demonstrated that control of Lp replication is NLRC4/NAIP5-dependent and likely mediated through regulation of pyroptosis [14]. Therefore increased growth of Lp in the lungs of Nlrc4-/and Nlrc4-/-/Tlr5-/- mice may be due to enhanced replication or failure of alveolar macrophage to undergo pyroptosis in NLRC4-deficient lungs. Alternatively elevated CFUs in Nlrc4-/- and Nlrc4-/-/Tlr5-/- mice could also be explained by enhanced levels of IL-1 $\beta$ or IL-18 in WT animals. Although we found no difference in IL- $1 \beta$ production in lungs of Lp-infected mice WT mice compared to Nlrc4-/- and Nlrc4-/-/Tlr5-/- [38]. Transcriptional analysis of the array data reveal that IL-1 $\beta$ is transcriptionally increased at similar levels in both animals, our methods, which involve measurement of cytokines in lung homogenates, may not adequately distinguish cleaved bioactive IL-1 $\beta$ from pro-IL-1 $\beta$ Nlrc4 $-/$ - and WT animals infected by Lp, suggesting that early induction of pro-IL-1 $\beta$ is found in both WT and Nlrc4-/animals. In support of a possible IL-18-dependent mechanism, a previous study demonstrated that IL-18 blockade with monoclonal antibodies contributes to control of $\mathrm{Lp}$ in the lungs when IL-12 is concurrently blocked, likely through an IFN- $\gamma$ dependent mechanism [35]. However, others have found that $I l 1 \beta-/-/ I l 18-/-$ mice are not more susceptible to Lp intraperitoneal infection when compared to WT mice [39]. Such a mechanism could contribute to NLRC4-dependent regulation of Lp growth during pulmonary infection.

In addition our study reveals elevated TNF and CXCL2 at 24 hours post-infection in Nlrc4-/-/Tlr5-/- mice compared to Nlrc4-/- and WT mice. The mechanisms underlying cytokine suppression seen in Nlrc4-/- animals compared to Nlrc4-/-/Tlr5-/- animals are currently only speculative. Perhaps signaling through TLR5 may evoke an anti-inflammatory response by causing refractory signaling in TLR signaling pathways at later timepoints $(24 \mathrm{~h})$. Another explanation may be that early TLR5mediated neutrophil recruitment seen in WT mice may help to contain the inflammatory response either by eliciting an anti-inflammatory response (for example, by eferocytosis of apoptotic PMNs [40]) or by clearing a pro-inflammatory stimulus (presumably by phagoctosis).

We also observed that NLRC4-deficient animals have increased alveolar space inflammation at $72 \mathrm{~h}$ postinfection. One possible explanation for these findings is that failure to control Lp replication in alveolar macrophages of Nlrc4-/- animals contributes to delayed pulmonary clearance of Lp. The higher bacterial burden would therefore enhance later recruitment of lung inflammation by CFU-dependent increases in signaling through other pathways (such as Myd88-dependent pathways) known to be activated by Lp [5]. At 72 hours, the increase in inflammatory cells seen histologically in the airways of Nlrc4-/- mice is different from the bronchoalveolar lavage data that has no difference. The likely explanation for this difference is that the BAL fluid samples cells that are easily recovered from the conducting airways and the alveolar lumina, while the histology measures the aggregates of inflammatory cells that occupy the alveoli and may not be readily dislodged and sampled during lavage. Our findings are consistent with a delayed Lp clearance model with increased bacterial loads even at early time points in Nlrc4-/- mice compared to WT.

In our study we show that growth of Lp in alveolar macrophages is NLRC4-dependent and TLR5-independent, and provides a plausible cellular mechanism for the increased in vivo susceptibility of Nlrc4-/- mice to Lp. Murine alveolar macrophages differ from other primary and BMDMs in their ability to sense flagellin in a TLR5dependent manner [4]. Despite these unique features of TLR5 in alveolar macrophages, our data show that control of $\mathrm{Lp}$ replication is not influenced by the presence of TLR5. Furthermore, our observations suggest that flagellin induces NLRC4-mediated resistance to Lp in murine alveolar macrophages, as has been described for BMDMs $[10,11,15,16,19,20]$. The implications of these data for human biology are not clear. Previous work has shown that human alveolar macrophages are more permissive to Lp replication than blood-derived monocytes [41]. Moreover, human alveolar macrophages differ from monocytes in the requirements for activation of the NLRC4 inflammasome. Alveolar macrophages lack constitutively activated caspase- 1 and require a second stimulus for IL- $1 \beta$ secretion in response to TLR ligands, in contrast to monocytes [42-44]. Lp growth in human alveolar macrophages is at least partially NLRC4 and NAIP-dependent as determined by enhancement of Lp growth by siRNA knockdown of NLRC4 or NAIP in vitro [45]. Furthermore Lp has been shown to enhance the transcription of $\beta$-defensin-3 in a TLR5-dependent manner, which may influence Lp replication in humans differently from the mouse $[46,47]$. Whether TLR5 knockdown regulates replication of Lp in human AMs is unknown. The precise molecular mechanism of NLRC4 and NAIP control in the both murine and human alveolar macrophage is the focus of current research efforts.

Messenger RNA arrays performed on genes expressed in whole lung showed no difference between Nlrc4-/and WT animals suggesting that NLRC4 inflammasome signaling at 4 hours is independent of transcription and may occur by post-translational mechanisms. After recognition of flagellin, the NLRC4/NAIP5 inflammasome oligomerizes and activates a proteolytic cascade beginning with activation of caspase-1. The lack of transcriptional regulation of NAIP5/NLRC4 inflammasome accompanied 
by the activation of proteolytic cascades by Lp has been observed previously at 4 hours in vitro in BMDMs isolated from $\mathrm{C} 57 \mathrm{Bl} / 6$ mice compared to the $\mathrm{A} / \mathrm{J}$ strain [48]. A separate study demonstrated that functionally deficient NAIP derived from $\mathrm{A} / \mathrm{J}$ mice may regulate transcriptional signaling through IRF1 and IRF8 [49]. Due to experimental differences, it is difficult to compare our data with these previous findings (bone marrow derived macrophages infected ex vivo with Lp versus whole lung mRNA after in vivo aerosol infection). Our study is also potentially limited by power, and it is possible that significant differences in the transcriptome might be obscured by the small sample size. The absence of mRNA differences seen in vivo suggest that the observed phenotype is from posttranslational, caspase- 1 dependent events such as pyroptosis. Induction of NLRC4-mediated pyroptosis by Lp may enhance bacterial release by susceptible macrophages and permit phagocytosis by newly recruited neutrophils. This may promote bacterial clearance in the alveolar space, as seen in other models of intracellular infection [39]. Whether pyroptosis contributes to resistance to pneumonic legionellosis is currently unknown.

\section{Conclusions}

These studies reveal that NLRC4 and TLR5 control different responses to Lp flagellin in an aerosolized murine infection model and NLRC4 regulates replication in both lungs and alveolar macrophages.

\section{Additional files}

Additional file 1: Table S1. Genes differentially expressed in WT lungs after aerosol infection with $\mathrm{Lp}$. Genes in this list had differentially expressed mRNA in WT Lp-infected lungs compared to uninfected controls. $\log _{2}$ fold change was determined by the averaged difference between the $\log _{2}$ mRNA values in lungs infected with $L p$ (WTstim) and uninfected lungs (WTunstim). Average expression in WTstim and WTunstim is the average of $\log _{2}$ transformed mRNA expression values in two mice. Genes named "—" had no identifiers in probeset. Probe identifiers are from Affymetrix 2.0 array designations. Unadjusted $p$ values were determined as described in methods. Adjusted $\mathrm{p}$ values were determined using a false discovery rate of $5 \%$ in the method of Benjamini and Hochberg (see methods).

Additional file 2: Table S2. Genes differentially expressed in N/rc4-/lungs after aerosol infection with Lp. Genes in this list had differentially expressed mRNA in N/rc4-/- Lp-infected lungs compared to uninfected N/rc4-/- controls. $\log _{2}$ fold change was determined by the averaged difference between the $\log _{2}$ mRNA values in lungs infected with Lp (N/rc4-/- ${ }_{\text {stim }}$ ) and uninfected lungs (N/rc4-/-unstim). Average expression in Nirc4-/- stim and N/rc4-/-unstim is the average of $\log _{2}$ transformed mRNA expression values in two mice. Genes named "- - had no naming identfication. Probe identifiers are from Affymetrix 2.0 array designations. Unadjusted $p$ values were determined as described in methods. Adjusted $p$ values were determined using a false discovery rate of $5 \%$ in the method of Benjamini and Hochberg (see methods).

Additional file 3: Table S3. Genes signficantly decreased in WT Lp-infected lungs but not significant in N/rc4-/- mice.

Additional file 4: Table S4. Genes signficantly increased in WT Lp-infected lungs but not significant in N/rc4-/- mice.

\section{Abbreviations}

AM: Alveolar macrophage; ASC: Apoptosis-associated speck protein containing caspase recruitment domain; BCYE: Buffered charcoal yeast extract; BMDM: Bone marrow derived macrophages; CARD: Caspase recruitment domain; CFU: Colony forming unit; Cl: Confidence interval; CXCL: Chemokine (C-X-C motif) ligand; CCL: Chemokine (C-C motif) ligand; FlaA-: Flagellin deficient; Lp: Legionella pneumophila; MOl: Multiplicity of infection; NAIP: Neuronal apoptosis inhibitory protein; NLR: Nucleotidebinding oligomerization domain-like receptor; NLRC4: Nucleotide-binding oligomerization domain containing-like receptor family, caspase recruitment domain (CARD) containing 4; NOD: Nucleotide-binding oligomerization domain; RIKEN: Rikagaku Kenkyusho; RMA: Robust multichip average; SEM: Standard error of mean; siRNA: Small inhibitory RNA; WT: Wild-type.

\section{Competing interests}

The authors declare that they have no competing interests.

\section{Authors' contributions}

WRB conceived and designed the study, carried out the molecular array studies, performed the statistical analysis, and drafted the manuscript. SJS conceived and designed the study and performed the in vivo infection, studies, and edited the manuscript. KDS examined the lung histology and edited the manuscript. TRH conceived and designed the study and helped with drafting the manuscript and statistical analysis. All authors read and approved the final manuscript.

\section{Acknowledgements}

We thank Shizuo Akira and Vishva Dixit for the mouse strains and Klaus Heuner for the Legionella strains. We thank Dr. Alan Aderem from Seattle BioMed for advice and David Rodriguez for technical assistance. We thank the following individuals from the University of Washington for their assistance: Glenna Peterson, Rick Wells, Malinka Jansson-Hutson, Ravi lyer, and Michelle Timko.

This work was supported by the National Institute of Allergy and Infectious Diseases at the National Institutes of Health [K08 Al080952 to WRB,

1K24Al089794 to TRH, 1R01Al062859 to KDS, and R01 Al093646 to SJS].

Received: 19 March 2013 Accepted: 8 August 2013

Published: 10 August 2013

\section{References}

1. Benin AL, Benson RF, Besser RE: Trends in legionnaires disease, 1980-1998: declining mortality and new patterns of diagnosis. Clin Infect Dis 2002, 35(9):1039-1046

2. Lau HY, Ashbolt NJ: The role of biofilms and protozoa in Legionella pathogenesis: implications for drinking water. Journal of applied microbiology 2009, 107(2):368-378.

3. Archer KA, Roy CR: MyD88-dependent responses involving toll-like receptor 2 are important for protection and clearance of Legionella pneumophila in a mouse model of Legionnaires' disease. Infect Immun 2006, 74(6):3325-3333.

4. Hawn TR, Berrington WR, Smith IA, Uematsu S, Akira S, Aderem A, Smith KD, Skerrett SJ: Altered inflammatory responses in TLR5-deficient mice infected with Legionella pneumophila. J Immunol 2007, 179(10):6981-6987.

5. Hawn TR, Smith KD, Aderem A, Skerrett SJ: Myeloid differentiation primary response gene (88)- and toll-like receptor 2-deficient mice are susceptible to infection with aerosolized Legionella pneumophila. J Infect Dis 2006, 193(12):1693-1702.

6. Archer KA, Alexopoulou L, Flavell RA, Roy CR: Multiple MyD88-dependent responses contribute to pulmonary clearance of Legionella pneumophila. Cell Microbiol 2009, 11(1):21-36.

7. Monroe KM, McWhirter SM, Vance RE: Identification of host cytosolic sensors and bacterial factors regulating the type I interferon response to Legionella pneumophila. PLOS pathogens 2009, 5(11):e1000665.

8. Frutuoso MS, Hori Jl, Pereira MS, Junior DS, Sonego F, Kobayashi KS, Flavell RA, Cunha FQ, Zamboni DS: The pattern recognition receptors Nod1 and Nod2 account for neutrophil recruitment to the lungs of mice infected with Legionella pneumophila. Microbes Infect 2010, 12(11):819-827.

9. Berrington WR, lyer R, Wells RD, Smith KD, Skerrett SJ, Hawn TR: NOD1 and NOD2 regulation of pulmonary innate immunity to Legionella pneumophila. Eur J Immunol 2010, 40(12):3519-3527. 
10. Zamboni DS, Kobayashi KS, Kohlsdorf T, Ogura Y, Long EM, Vance RE, Kuida K, Mariathasan S, Dixit VM, Flavell RA, et al: The birc1e cytosolic patternrecognition receptor contributes to the detection and control of Legionella pneumophila infection. Nat Immunol 2006, 7(3):318-325.

11. Ren T, Zamboni DS, Roy CR, Dietrich WF, Vance RE: Flagellin-deficient Legionella mutants evade caspase-1- and Naip5-mediated macrophage immunity. PLoS pathogens 2006, 2(3):e18.

12. Kikuchi T, Kobayashi T, Gomi K, Suzuki T, Tokue Y, Watanabe A, Nukiwa T: Dendritic cells pulsed with live and dead Legionella pneumophila elicit distinct immune responses. J Immunol 2004, 172(3):1727-1734.

13. Khare S, Dorfleutner A, Bryan NB, Yun C, Radian AD, de Almeida L, Rojanasakul Y, Stehlik C: An NLRP7-containing inflammasome mediates recognition of microbial lipopeptides in human macrophages. Immunity 2012, 36(3):464-476

14. Lightfield KL, Persson J, Brubaker SW, Witte CE, von Moltke J, Dunipace EA, Henry T, Sun YH, Cado D, Dietrich WF, et al: Critical function for Naip5 in inflammasome activation by a conserved carboxy-terminal domain of flagellin. Nat Immuno/ 2008, 9(10):1171-1178.

15. Molofsky AB, Byrne BG, Whitfield NN, Madigan CA, Fuse ET, Tateda K, Swanson MS: Cytosolic recognition of flagellin by mouse macrophages restricts Legionella pneumophila infection. Journal of Experimental Medicine 2006, 203(4):1093-1104.

16. Amer A, Franchi L, Kanneganti TD, Body-Malapel M, Ozoren N, Brady G, Meshinchi S, Jagirdar R, Gewirtz A, Akira S, et al: Regulation of Legionella phagosome maturation and infection through flagellin and host Ipaf. J Biol Chem 2006, 281(46):35217-35223.

17. Diez E, Lee SH, Gauthier S, Yaraghi Z, Tremblay M, Vidal S, Gros P: Birc1e is the gene within the Lgn1 locus associated with resistance to Legionella pneumophila. Nat Genet 2003, 33(1):55-60.

18. Growney JD, Dietrich WF: High-resolution genetic and physical map of the Lgn1 interval in C57BL/6J implicates Naip2 or Naip5 in Legionella pneumophila pathogenesis. Genome Res 2000, 10(8):1158-1171.

19. Kofoed EM, Vance RE: Innate immune recognition of bacterial ligands by NAIPs determines inflammasome specificity. Nature 2011, 477(7366):592-595.

20. Zhao Y, Yang J, Shi J, Gong YN, Lu Q, Xu H, Liu L, Shao F: The NLRC4 inflammasome receptors for bacterial flagellin and type III secretion apparatus. Nature 2011, 477(7366):596-600.

21. Case CL, Shin S, Roy CR: Asc and Ipaf Inflammasomes direct distinct pathways for caspase-1 activation in response to Legionella pneumophila. Infect Immun 2009, 77(5):1981-1991.

22. Pereira MS, Morgantetti GF, Massis LM, Horta CV, Hori Jl, Zamboni DS: Activation of NLRC4 by flagellated bacteria triggers caspase-1-dependent and -independent responses to restrict Legionella pneumophila replication in macrophages and in vivo. J Immunol 2011, 187(12):6447-6455.

23. Brieland J, Freeman P, Kunkel R, Chrisp C, Hurley M, Fantone J, Engleberg C: Replicative Legionella pneumophila lung infection in intratracheally inoculated A/J mice. A murine model of human Legionnaires' disease. Am J Pathol 1994, 145(6):1537-1546.

24. Molofsky AB, Shetron-Rama LM, Swanson MS: Components of the Legionella pneumophila flagellar regulon contribute to multiple virulence traits, including lysosome avoidance and macrophage death. Infect Immun 2005, 73(9):5720-5734

25. Hammer BK, Tateda ES, Swanson MS: A two-component regulator induces the transmission phenotype of stationary-phase Legionella pneumophila. Mol Microbiol 2002, 44(1):107-118

26. Dietrich C, Heuner K, Brand BC, Hacker J, Steinert M: Flagellum of Legionella pneumophila positively affects the early phase of infection of eukaryotic host cells. Infect Immun 2001, 69(4):2116-2122.

27. Uematsu S, Jang MH, Chevrier N, Guo Z, Kumagai Y, Yamamoto M, Kato H,

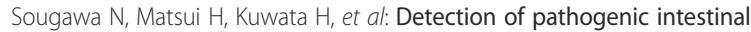
bacteria by Toll-like receptor 5 on intestinal CD11 + lamina propria cells. Nat Immunol 2006, 7(8):868-874

28. Mariathasan S, Newton K, Monack DM, Vucic D, French DM, Lee WP, RooseGirma M, Erickson S, Dixit VM: Differential activation of the inflammasome by caspase-1 adaptors ASC and Ipaf. Nature 2004, 430(6996):213-218.

29. Skerrett SJ, Liggitt HD, Hajiar AM, Wilson CB: Cutting edge: myeloid differentiation factor 88 is essential for pulmonary host defense against Pseudomonas aeruginosa but not Staphylococcus aureus. J Immunol 2004, 172(6):3377-3381.

30. Skerrett SJ, Henderson WR, Martin TR: Alveolar macrophage function in rats with severe protein calorie malnutrition. Arachidonic acid metabolism, cytokine release, and antimicrobial activity. J Immunol 1990, 144(3):1052-1061.
31. Irizarry RA, Hobbs B, Collin F, Beazer-Barclay YD, Antonellis KJ, Scherf U, Speed TP: Exploration, normalization, and summaries of high density oligonucleotide array probe level data. Biostatistics 2003, 4(2):249-264.

32. Carey VJ, Gentry J, Whalen E, Gentleman R: Network structures and algorithms in bioconductor. Bioinformatics 2005, 21(1):135-136

33. Smyth GK: Linear models and empirical bayes methods for assessing differential expression in microarray experiments. Statistical applications in genetics and molecular biology 2004, 3(Article3):1-25.

34. Benjamini $Y$, Hochberg $Y$ : Controlling the false discovery rate: a practical and powerful approach to multiple testing. J R Statist Soc B 1995, 57(1):289-300.

35. Brieland JK, Jackson C, Hurst S, Loebenberg D, Muchamuel T, Debets R, Kastelein R, Churakova T, Abrams J, Hare R, et al: Immunomodulatory role of endogenous interleukin-18 in gamma interferon-mediated resolution of replicative Legionella pneumophila lung infection. Infect Immun 2000, 68(12):6567-6573.

36. Subramanian A, Tamayo P, Mootha VK, Mukherjee S, Ebert BL, Gillette MA, Paulovich A, Pomeroy SL, Golub TR, Lander ES, et al: Gene set enrichment analysis: a knowledge-based approach for interpreting genome-wide expression profiles. Proc Natl Acad Sci U S A 2005, 102(43):15545-15550.

37. Wettenhall JM, Smyth GK: limmaGUI: a graphical user interface for linear modeling of microarray data. Bioinformatics 2004, 20(18):3705-3706.

38. Rogers J, Hakki A, Perkins I, Newton C, Widen R, Burdash N, Klein T, Friedman $\mathrm{H}$ : Legionella pneumophila infection up-regulates dendritic cell toll-like receptor 2 (TLR2)/TLR4 expression and key maturation markers. Infect Immun 2007, 75(6):3205-3208.

39. Miao EA, Leaf IA, Treuting PM, Mao DP, Dors M, Sarkar A, Warren SE, Wewers MD, Aderem A: Caspase-1-induced pyroptosis is an innate immune effector mechanism against intracellular bacteria. Nat Immunol 2010, 11(12):1136-1142.

40. McDonald PP, Fadok VA, Bratton D, Henson PM: Transcriptional and translational regulation of inflammatory mediator production by endogenous TGF-beta in macrophages that have ingested apoptotic cells. J Immunol 1999, 163(11):6164-6172.

41. Park DR, Skerrett SJ: IL-10 enhances the growth of Legionella pneumophila in human mononuclear phagocytes and reverses the protective effect of IFN- $\gamma$ : differential responses of blood monocytes and alveolar macrophages. J Immunol 1996, 157(6):2528-2538.

42. Wewers MD, Dare HA, Winnard AV, Parker JM, Miller DK: IL-1 betaconverting enzyme (ICE) is present and functional in human alveolar macrophages: macrophage IL-1 beta release limitation is ICE independent. J Immunol 1997, 159(12):5964-5972.

43. Li H, Willingham SB, Ting JP, Re F: Cutting edge: inflammasome activation by alum and alum's adjuvant effect are mediated by NLRP3. J Immunol 2008, 181(1):17-21.

44. Netea MG, Nold-Petry CA, Nold MF, Joosten LA, Opitz B, van der Meer JH, van de Veerdonk FL, Ferwerda G, Heinhuis B, Devesa I, et al: Differential requirement for the activation of the inflammasome for processing and release of IL-1beta in monocytes and macrophages. Blood 2009, 113(10):2324-2335.

45. Vinzing M, Eitel J, Lippmann J, Hocke AC, Zahlten J, Slevogt H, N'Guessan PD, Gunther S, Schmeck B, Hippenstiel S, et al: NAIP and Ipaf control Legionella pneumophila replication in human cells. J Immunol 2008, 180(10):6808-6815.

46. Scharf S, Hippenstiel S, Flieger A, Suttorp N, N'Guessan PD: Induction of human beta-defensin- 2 in pulmonary epithelial cells by Legionella pneumophila: involvement of TLR2 and TLR5, p38 MAPK, JNK, NFkappaB, and AP-1. American journal of physiology Lung cellular and molecular physiology 2010, 298(5):L687-L695.

47. Scharf S, Vardarova K, Lang F, Schmeck B, Opitz B, Flieger A, Heuner K, Hippenstiel S, Suttorp N, N'Guessan PD: Legionella pneumophila induces human beta defensin-3 in pulmonary cells. Respiratory research 2010, 11:93.

48. Fortier A, Faucher SP, Diallo K, Gros P: Global cellular changes induced by Legionella pneumophila infection of bone marrow-derived macrophages. Immunobiology 2011, 216(12):1274-1285.

49. Fortier A, Doiron K, Saleh M, Grinstein S, Gros P: Restriction of Legionella pneumophila replication in macrophages requires concerted action of the transcriptional regulators Irf1 and Irf8 and nod-like receptors Naip5 and Nirc4. Infect Immun 2009, 77(11):4794-4805.

doi:10.1186/1471-2334-13-371

Cite this article as: Berrington et al:: Nucleotide-binding oligomerization domain containing-like receptor family, caspase recruitment domain (CARD) containing 4 (NLRC4) regulates intrapulmonary replication of aerosolized Legionella pneumophila. BMC Infectious Diseases 2013 13:371. 